\title{
Storing entanglement of nuclear spins via Uhrig Dynamical Decoupling
}

\author{
Soumya Singha Roy ${ }^{1}$, T. S. Mahesh ${ }^{1}$ and G. S. Agarwal ${ }^{2}$ * \\ ${ }^{1}$ Indian Institute of Science Education and Research, Pune 411008, India \\ ${ }^{2}$ Department of Physics, Oklahoma State University, Stillwater, OK 74078, USA
}

(Dated: June 7, 2018)

\begin{abstract}
Stroboscopic spin flips have already been shown to prolong the coherence times of quantum systems under noisy environments. Uhrig's dynamical decoupling scheme provides an optimal sequence for a quantum system interacting with a dephasing bath. Several experimental demonstrations have already verified the efficiency of such dynamical decoupling schemes in preserving single qubit coherences. In this work we describe the experimental study of Uhrig's dynamical decoupling in preserving two-qubit entangled states using an ensemble of spin-1/2 nuclear pairs in solution state. We find that the performance of odd-order Uhrig sequences in preserving entanglement is superior to both even-order Uhrig sequences and periodic spin-flip sequences. We also find that there exists an optimal order of the Uhrig sequence using which a singlet state can be stored at high correlation for about 30 seconds.
\end{abstract}

PACS numbers: 03.65.Yz, 03.67.Bg, 03.67.Pp, 76.60.-k

Keywords: Quantum information, quantum coherence, uhrig dynamical decoupling, nuclear magnetic resonance, singlet-states, long-lived states, Bell states

\section{INTRODUCTION}

Harnessing the quantum properties of physical systems has several potential applications, particularly in information processing, secure data communications, and quantum simulators [1]. It is believed that such quantum devices may play an important role in future technology [2]. But their physical realization is challenging mainly because of decoherence - the decay of the coherent states due to interaction with the surrounding environment [3, 4]. Therefore it is important to minimize the effects of decoherence using suitable perturbation on the quantum system [5]. One technique known as 'dynamical decoupling' involves protecting the quantum state from decoherence by driving the system in a systematic manner such that the effective interactions with the environment at different instants of time cancel one another.

Preserving nuclear spin coherences by spin flips at regular intervals was long been known in nuclear magnetic resonance (NMR) as the famous Carr-Purcell-MeiboomGill (CPMG) sequence [6, 7]. The CPMG sequence is widely used in NMR to measure the transverse relaxation time constants in the presence of spatial inhomogeneity of the static magnetic field and temporal fluctuations in the local fields arising due to molecular motion [8]. The sequence involves a set of $\mathrm{N} \pi$ pulses uniformly distributed in a duration $[0, T]$ at time instants $\left\{t_{1}, t_{2}, \cdots, t_{N}\right\}$. Assuming instantaneous $\pi$ pulses, $j^{\text {th }}$ time instant is linear in $j$

$$
t_{j}^{\mathrm{CPMG}}=T\left(\frac{2 j-1}{2 N}\right) .
$$

Of course, in practice the $\pi$ pulses do have finite duration owing to the limited power of electromagnetic ir- radiation generated by a given hardware. Further, the constant time period between these spin flips should ideally be shorter than the correlation time of the spin-bath interaction. Even this delay is limited by the maximum duty-cycle that is allowed for the hardware. Dynamical decoupling with such bounded controls have also been suggested $9-12$. For instance Hao et al. have been able to calculate, using a particular type of atomic systems, the maximum delay between spin-flips in order to efficiently suppress decoherence due to a bath with a finite cut-off frequency [13]. By studying the efficiency of the decoupling as a function of the CPMG period often it is possible to extract valuable informations about molecular dynamics and such studies are broadly categorized under 'CPMG dispersion' experiments [14].

Uhrig generalized the CPMG sequence by considering an optimal distribution $\left\{t_{1}, t_{2}, \cdots, t_{N}\right\}$ of $N$ spin flips in a given duration $[0, T]$ of time that provides most efficient dynamical decoupling [15]. Using a simple dephasing model, Uhrig proved that the time instants should vary as a squared sine bell:

$$
t_{j}=T \sin ^{2}\left(\frac{\pi j}{2 N+2}\right)
$$

UDD works well in systems having a high-frequency dominated bath with a sharp cutoff [16 18]. On the other hand when the spectral density of the bath has a soft cutoff (such as a broad Gaussian or Lorentzian), the CPMG sequence was found to outperform the UDD sequence [19 25]. Suter and co-workers have studied these different regimes and arrived at optimal conditions for the dynamical decoupling [26].

Recently Agarwal has shown using theoretical and numerical calculations that even entangled states of twospin systems can be stored more efficiently using UDD 27]. Since entangled states play a central role in QIP, teleportation, data encryption, and so on, saving entan-

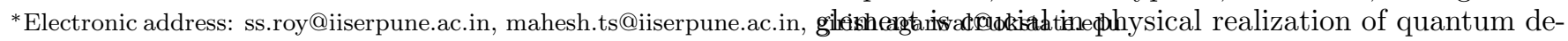




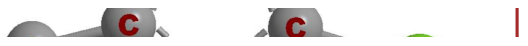

FIG. 1: (Color online) The ${ }^{1} \mathrm{H}$ NMR spectrum and the molecular structure of 5-chlorothiophene-2-carbonitrile.

vices 11. More recently dynamical decoupling on an electron-nuclear spin-pair in a solid state system has been shown to prolong the pseudoentanglement lifetime by two orders of magnitude [28].

While much of the experimental effort has been on testing the loss of coherence due to $\mathrm{T}_{2}$ processes, ours is one of the first experiments where we study not only the loss of coherences, but also the loss of entanglement due to both $\mathrm{T}_{1}$ and $\mathrm{T}_{2}$ processes. Though newer sequences have been suggested to decouple both of these processes, these are yet to be studied experimentally [29, 30]. Since we have developed experimental techniques where we can prepare Bell states with high fidelity and characterize these states with high precision [31, 32], we explore the utility of different dynamical decoupling sequences on systems wherein both $T_{1}$ and $T_{2}$ relaxations are significant. In the following we describe preparation of Bell states using a two-qubit NMR system and then describe the comparative performances of CPMG and UDD on such entangled states.

\section{PREPARATION AND STORAGE OF ENTANGLEMENT}

We study storage of entanglement by dynamic decoupling on a pair of spin- $1 / 2$ nuclei using liquid state NMR techniques. The sample consisted of $5 \mathrm{mg}$ of 5 -chlorothiophene-2-carbonitrile dissolved in $0.75 \mathrm{ml}$ of dimethyl sulphoxide (see Figure 1). The two protons of the solute molecule differ in the Larmor frequency by $\Delta \nu=270.4 \mathrm{~Hz}$ and have an indirect spin-spin coupling constant of $J=4.1 \mathrm{~Hz}$. The $T_{2}$ relaxation time constants for the two protons are about $2.3 \mathrm{~s}$ and the $T_{1}$ relaxation time constants are about $6.3 \mathrm{~s}$. All the experiments are carried out in a Bruker $500 \mathrm{MHz}$ NMR spectrometer at an ambient temperature of $300 \mathrm{~K}$.

\section{Pseudopure States}

An ensemble of nuclear spin-systems in thermal equilibrium at room temperature $T$ exists in a mixed state at high spin temperature, since the Zeeman energy gaps $\Delta E$ are much smaller than the Boltzman energy $k T$. Therefore the general state of an ensemble of spin- $1 / 2$ nuclear pairs can be described by the density matrix

$$
\rho_{\mathrm{eq}}=\frac{1}{4} \mathbb{1}+\epsilon \rho_{\Delta},
$$

where the identity part is usually ignored and the traceless matrix $\rho_{\Delta}$ is called the deviation density matrix. The dimensionless quantity $\epsilon \sim \Delta E / k T$ and have magnitudes $\sim 10^{-4}$ for protons in currently available magnetic fields at ordinary room temperatures. Using a combination of unitary and non-unitary processes, it is possible to transform the above state to a pseudopure $|\psi\rangle$ state given by

$$
\rho_{\mathrm{pps}}=\frac{1}{4}(1-\epsilon) \mathbb{1}+\epsilon|\psi\rangle\langle\psi|
$$

33]. Here $\epsilon$ is a measure of the magnetization retained in the pseudopure state. All the kets in the rest of the article must be understood as pseudopure states.

\section{Preparation of pseudoentangled States}

High fidelity entangled states are prepared via long lived singlet states in a procedure described by Soumya et al [31, 32]. The method is briefly explained in the following for completeness.

The Hamiltonian for an ensemble of spin- $1 / 2$ nuclear pairs of same isotope, in the RF interaction frame, can be expressed as

$$
\mathcal{H}^{\mathrm{eff}}=h\left[\frac{\Delta \nu}{2} I_{z}^{1}-\frac{\Delta \nu}{2} I_{z}^{2}+J I^{1} \cdot I^{2}+\nu_{12} I_{x}^{1,2}\right] .
$$

Here $h \Delta \nu$ corresponds to the difference in the Zeeman energy gaps of the two spins (due to the chemical shift difference), $I_{z}^{k}$ corresponds to the spin angular momentum of $k^{\text {th }}$ spin, $J$ is the indirect spin-spin coupling, and $\nu_{12}$ is the amplitude of circularly polarized component of the radio frequency field, which is at resonance with the mean frequency of the two spins.

In the limiting case of $\Delta \nu \rightarrow 0$, the system is said to have magnetic equivalence, and the singlet state $\left|S_{0}\right\rangle=$ $(|01\rangle-|10\rangle) / \sqrt{2}$, and the triplet states $\left|T_{1}\right\rangle=|00\rangle,\left|T_{0}\right\rangle=$ $(|01\rangle+|10\rangle) / \sqrt{2}$, and $\left|T_{-1}\right\rangle=|11\rangle$ form an orthonormal eigenbasis of the resulting internal Hamiltonian [8]

$$
\mathcal{H}_{\mathrm{eq}}^{\mathrm{eff}}=h J I^{1} \cdot I^{2} .
$$

The equivalence Hamiltonian may be realized by suppressing the chemical shift $\Delta \nu$ using a radio frequency 'spin-lock' 35.

The long-lived nature of singlet states under the equivalence Hamiltonian can be used to prepare high-fidelity Bell states. The experiment involves preparing an incoherent mixture of singlet and triplet states

$$
\rho(0)=-\mathbf{I}^{1} \cdot \mathbf{I}^{2} \equiv\left|S_{0}\right\rangle\left\langle S_{0}|-| T_{0}\right\rangle\left\langle T_{0}\right|
$$

from the equilibrium state $I_{z}^{1}+I_{z}^{2}$ by using the pulse sequence shown in Figure 2 [34. After preparing $\rho(0)$, the Hamiltonian $\mathcal{H}_{\mathrm{eq}}^{\mathrm{eff}}$ is imposed using RF spin-lock. During the spin-lock $\left|T_{0}\right\rangle$ state rapidly equilibrates with the other triplet states. On the other hand, the decay constant of singlet state $\left|S_{0}\right\rangle$ during the spin-lock is much longer than the spin-lattice relaxation time constant $\left(T_{1}\right)$ (and hence the singlet state is known as a long-lived state) [35, 36]. The long-life is attributed to the fact that the 


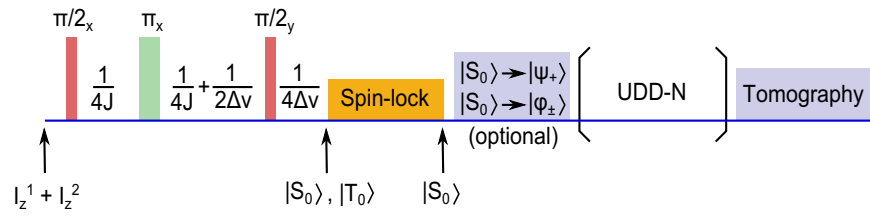

FIG. 2: (Color online) NMR pulse sequence to study dynamical decoupling on Bell states. An incoherent mixture of singlet and triplet states is prepared which under spin-lock purifies to singlet state. The resulting singlet state can be converted to other Bell states. Then dynamical decoupling sequence can be applied and the performance of the sequence can be studied by characterizing the residual state using density matrix tomography.

anti-symmetric singlet state can not be converted to symmetric triplet states via symmetry conserving relaxation mechanisms such as the intra-molecular dipolar interactions [37-39].

As a result of the long life-times under $\mathcal{H}_{\text {eq }}^{\text {eff }}$, the attenuated correlation of the singlet state

$$
\left\langle\mathbf{S}_{\mathbf{0}}\right\rangle=\frac{\operatorname{trace}\left[\left|S_{0}\right\rangle\left\langle S_{0}\right| \cdot \rho(t)\right]}{\sqrt{\operatorname{trace}\left[\rho^{2}(t)\right]}}
$$

with $\rho(t)$ improves over time $t$, reaches a maximum value and ultimately decays due to the symmetry breaking interactions [31]. The spin-lock imposing the Hamiltonian $\mathcal{H}_{\text {eq }}^{\text {eff }}$ can be turned off when the maximum correlation $\left\langle\mathbf{S}_{\mathbf{0}}\right\rangle$ is reached, and the high fidelity singlet state can be used for further studies.

Other Bell states can be obtained easily from the singlet state:

$$
\begin{aligned}
& \left|S_{0}\right\rangle \quad \stackrel{\mathrm{e}^{i \pi I_{z}^{1}}}{\longrightarrow} \quad\left|\psi_{+}\right\rangle=(|01\rangle+|10\rangle) / \sqrt{2}, \\
& \left|S_{0}\right\rangle \quad \stackrel{\mathrm{e}^{i \pi I_{x}^{1}}}{\longrightarrow} \quad\left|\phi_{-}\right\rangle=(|00\rangle-|11\rangle) / \sqrt{2}, \\
& \left|S_{0}\right\rangle \stackrel{\mathrm{e}^{i \pi I_{x}^{1} \cdot \mathrm{e}^{i \pi I_{z}^{1}}} \longrightarrow}{\longrightarrow}\left|\phi_{+}\right\rangle=(|00\rangle+|11\rangle) / \sqrt{2} .
\end{aligned}
$$

The $z$-rotation in the above propagators can be implemented by using chemical shift evolution for a period of $1 /(2 \Delta \nu)$, and qubit selective $x$-rotation can be implemented by using radio frequency pulses [32].

Details of dynamical decoupling on the Bell states will be described in the next sub-section. In order to investigate the decoupling performance one needs to quantify the decay of Bell states with decoupling duration. The Bell states by themselves are inaccessible to macroscopic observables, but can indirectly be detected transforming to observable single quantum coherences [35, 36]. Alternatively, a more detailed and quantitative analysis of Bell states may be carried out using density matrix tomography [31]. After measuring the density matrix, attenuated correlation can be evaluated for any Bell state using expressions similar to (8).

In the following we describe experimental implementations of dynamical decoupling after preparing such entangled states.

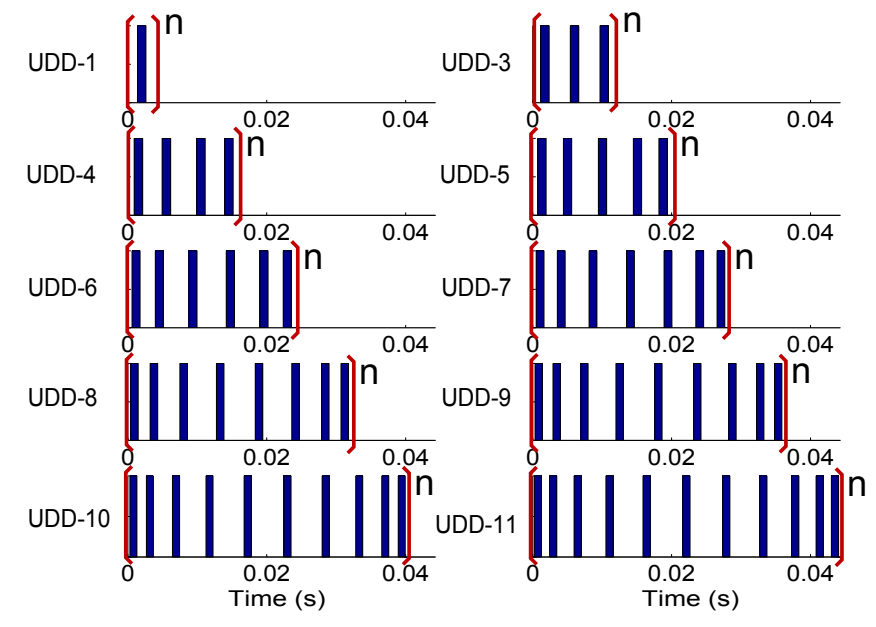

FIG. 3: (Color online) Pulse sequences for various orders of Uhrig Dynamical Decoupling. Note that both UDD-1 and UDD-2 are equivalent to CPMG. The time instants are calculated according to the expression (2), with $N$ being the order of UDD and the total period $T=N \times 4.0272 \mathrm{~ms}$.

\section{Dynamical Decoupling}

As described earlier, the UDD scheme consists of a sequence of spin flips placed at time instants given by the expression (2). Instead of applying the Uhrig's formula for the entire duration of decoupling, we apply the formula for a short time interval $(T)$ consisting of a small number $(N)$ of pulses and then repeat the sequence. Figure 3 shows pulse sequences for various orders of Uhrig Dynamical Decoupling (we refer to an N-pulse UDD sequence as UDD-N). Note that UDD-1 (and UDD-2) are equivalent to CPMG sequence, in which repeating segment consists of $\left[\tau_{\mathrm{CPMG}}-\pi-\tau_{\mathrm{CPMG}}\right]$. In our experiments, $\tau_{\mathrm{CPMG}}$ was set to $2 \mathrm{~ms}$ and the duration $\tau_{\pi}$ of the $\pi$ pulse was $27.2 \mu \mathrm{s}$. The total duration of UDD-N was set to $T=N\left(2 \tau_{\mathrm{CPMG}}+\tau_{\pi}\right)$, such that for an extended period of time, the total number of $\pi$ pulses remains same irrespective of the order of UDD. Only the distribution of $\pi$ pulses varies according to the order of UDD. For example in one second of decoupling, there will be about $250 \pi$ pulses in all UDD-N. Our investigation thus helps in studying the efficiency of decoupling over a fixed duration of time for a given number of $\pi$ pulses dispersed according to different orders of UDD.

Now we describe the performances of UDD-N on the singlet state which was prepared as explained before (see Figure 2). After applying UDD-N for a fixed duration of time, we carried out density matrix tomography and evaluated the correlation of the preserved state with theoretical singlet density matrix. The correlations for various orders of UDD are displayed in Figure 4. As can be seen from the figure, the singlet state can be preserved for longer durations by UDD-1 (CPMG) than no-decoupling. It is also clear that all even-order UDD sequences result in significant fluctuations in the correlation of the singlet 

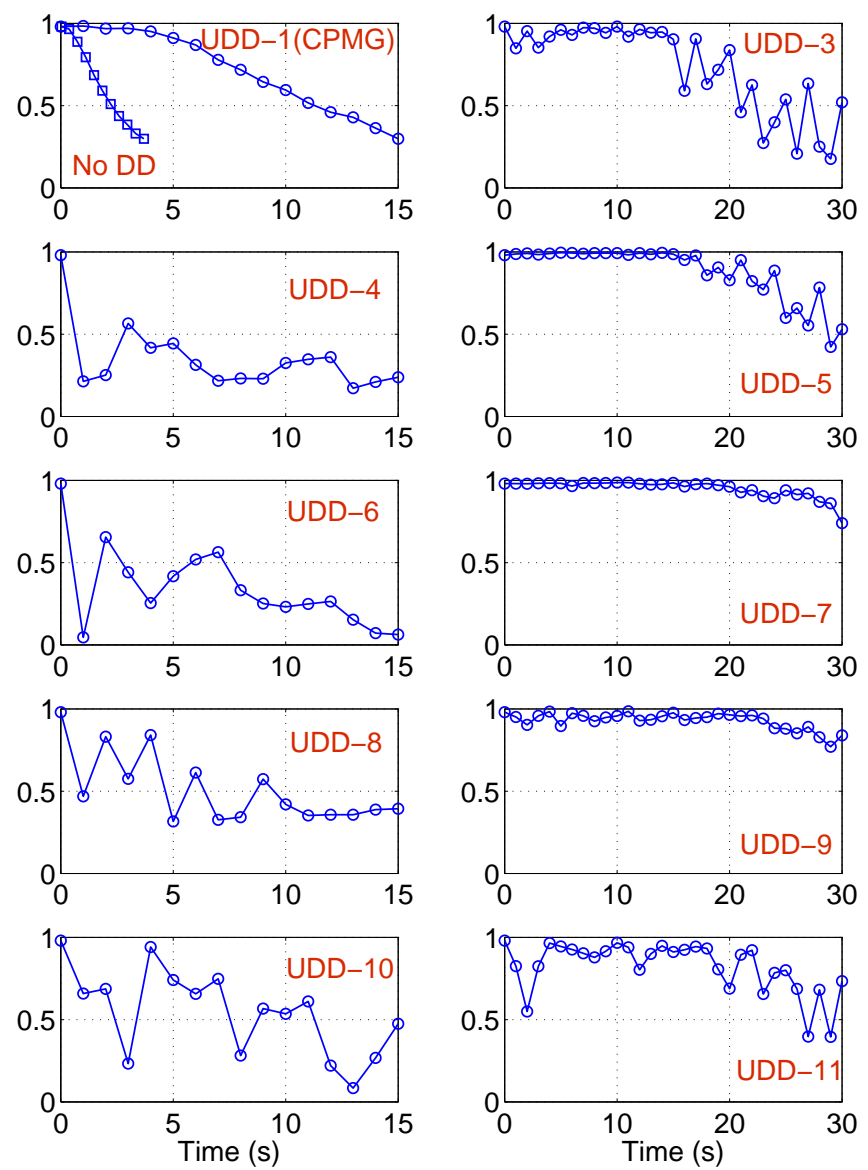

FIG. 4: (Color online) Experimental correlations (circles) of singlet state as a function of decoupling duration of various orders of UDD. Also shown in the top-left figure is the correlation decay under no dynamical decoupling (squares).

state. However, the odd order UDD preserve the singlet state for tens of seconds. For example, the correlation of the singlet state under UDD-7 at all the sampled time points till 20 seconds is above 0.96 . This rather surprising even-odd behavior is likely due to the differences in the performances of the even and odd ordered sequences against the spatial inhmongeneity of the RF pulses.

One way to quantify the efficiency of dynamical decoupling under various orders of UDD in figure 4, is by counting the number of time instants in which the correlation of the preserved state exceeds a given threshold. The bar plot in Figure 5 compares the number of time instants during decoupling under various orders of UDD in which the correlation of the singlet state exceeded 0.9. It can be seen that there exists an optimal order of UDD (for a given $\tau_{\mathrm{CPMG}}$ and $\tau_{\pi}$ ), which performs the most efficient decoupling. The optimality may be because of the finite width of the $\pi$ pulse. In a CPMG sequence the $\pi$ pulses are uniformly dispersed, while in Uhrig sequence the $\pi$ pulses are more crowded at the terminals (begin-

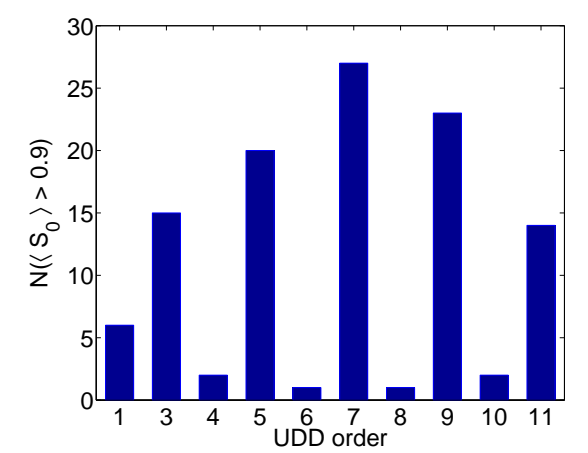

FIG. 5: (Color online) The number of time instants at which the correlation exceeded 0.9 for various orders of UDD-N.

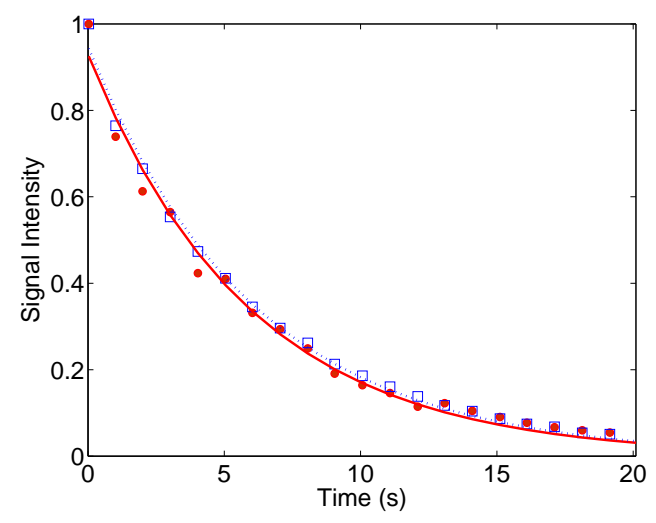

FIG. 6: (Color online) The decay of the singlet spin-order measured by converting it into observable single quantum magnetizations. The decay was studied under CPMG sequence (squares) as well as under Uhrig sequence (filled circles). The dashed and the solid line correspond to the exponential fits for CPMG and UDD-7 data points respectively.

ning and ending) of the sequence. For example, if there are too many $\pi$ pulses, Uhrig's formula will lead to an overlap of pulses. Experimentally, the overcrowding of $\pi$ pulses may also lead to RF heating of the sample and the probe. Thus the performance of the UDD sequence does not grow indefinitely with the order of the sequence, but instead will fall beyond a certain order. In our experimental setting, we find that UDD-7 is the optimal sequence for storing the singlet state. There are recent suggestions for decoupling using finite pulses, however these are yet to be studied experimentally [40, 41].

It can be noticed that the attenuated correlation (expression (8) ) is insensitive to the decay of the overall magnetization ( $\epsilon$ in (40), but simply measures the overlap between $\rho_{\Delta}$ and the theoretical density matrix $|\psi\rangle\langle\psi|$. An alternate method is to monitor the decay of magnetization (i.e., $\epsilon$ ) under dynamical decoupling. As already mentioned, singlet state itself can not be measured directly, but can be converted to observable magnetization by using a chemical shift evolution for a duration 

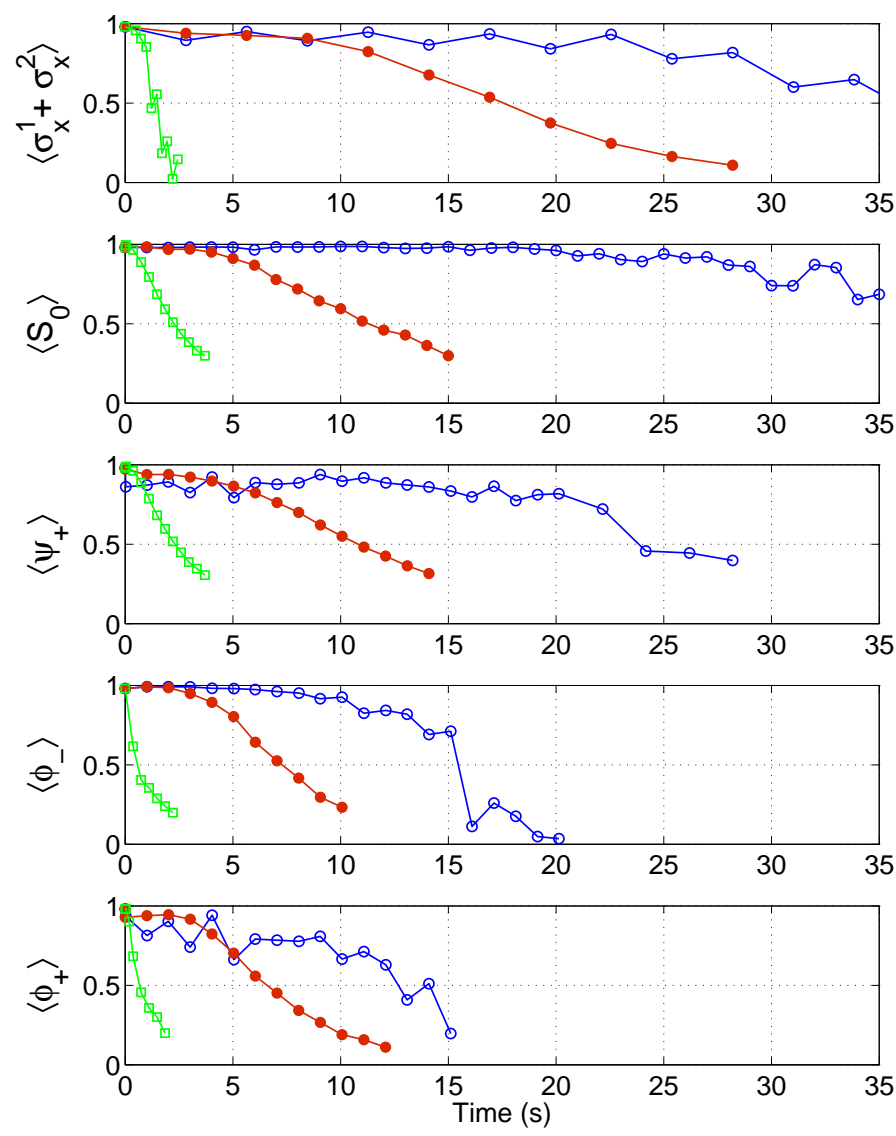

FIG. 7: (Color online) Experimental correlations of the product state and various Bell states as a function of duration under (i) no decoupling (open squares), (ii) CPMG sequence (filled circles), and (iii) UDD-7 (open circles).

$1 /(4 \Delta \nu)$ followed by a $\left(\frac{\pi}{2}\right)_{x(y)}$ pulse. Intensity of the resulting signal as a function of the duration of dynamical decoupling is shown in Figure 6. As can be seen, UDD-7 is no better than CPMG in preserving the overall spinorder. In fact the decay constant for CPMG and UDD-7 are $6.1 \mathrm{~s}$ and $5.9 \mathrm{~s}$ respectively.

Now we compare the efficiency of the optimal sequence UDD-7 with UDD-1 (CPMG) for preserving product state $\left(\sigma_{x}^{1}+\sigma_{x}^{2}\right)$ and other Bell states. Figure 7 shows the variation of correlation of product states and the Bell states as a function of the decoupling duration. Here, after preparing each of the initial state, the dynamical decoupling was applied for a fixed duration of time. To monitor the correlation, we have carried out the density matrix tomography as described earlier [31]. In the case of no decoupling, we observe a rapid decay of the correlation. The UDD-1 (CPMG) sequence shows some improvement in the storage time. However, UDD-7 clearly exhibits much longer storage times than the CPMG sequence. The superior performance of UDD-7 on the singlet state compared to other Bell states is presumably because of its antisymmetric property described in section II.

\section{CONCLUSIONS}

We have studied the efficiencies of CPMG as well as Uhrig dynamical decoupling sequences on 2-qubit Bell states both in terms of magnetization as well as in terms of correlation decay. While the Uhrig sequence is no better than CPMG sequence in terms of preserving the overall magnetization (or spin order), it clearly outperforms the CPMG sequence in preserving the correlation of the entangled as well as non-entangled states. We summarize three important features: (i) the even-order UDD sequences result is fluctuations in correlations, (ii) the odd-order UDD sequences out-perform the CPMG sequence, and (iii) there exists an optimal length for the odd order UDD sequence which exhibits most efficient decoupling. In our case, UDD-7 of $28.2 \mathrm{~ms}$ duration appeared to outperform all other sequences of both lower and higher orders. We are carrying out investigations into the effects of other experimental issues like RF inhomogeneity, resonance off-set, errors in calibration of pulse angle etc. These considerations may help in the theoretical and practical understanding of the optimal decoupling schemes.

\section{Acknowledgments}

GSA thanks Director, IISER-Pune for the invitation to work at the Institute which led to this collaboration. TSM acknowledges useful discussions with Prof. Dieter Suter, Prof. Anil Kumar, and Dr. Karthik Gopalakrishnan. The use of $500 \mathrm{MHz}$ NMR spectrometer at NMR Research Center, IISER-Pune is acknowledged.
[1] M. A. Nielsen and I. L. Chuang, Quantum Computation and Quantum Information, Cambridge University Press (2002).

[2] J.P.Dowling and G.J.Milburn, Phil. Trans. R. Soc. A 361, 3655 (2003)

[3] E. Joos, H. D. Zeh, C. Kiefer, D. J. W. Giulini, J. Kupsch, I. O. Stamatescu, Decoherence and the Appear- ance of a Classical World in Quantum Theory, 2nd Edn., Springer (2003).

[4] M. A. Schlosshauer, Decoherence: and the Quantum-ToClassical Transition, 2nd Edn. Springer (2007).

[5] L. Viola, E. Knill, and S. Lloyd, Phys. Rev. Lett. 82, 2417 (1999).

[6] H. Y. Carr and E. M. Purcell, Phys. Rev. 94, 630 (1954). 
[7] S. Meiboom and D. Gill, Rev. Sci. Instr. 29, 688 (1958).

[8] M. H. Levitt, Spin Dynamics, J. Wiley and Sons Ltd., Chichester (2002).

[9] L. Viola and E. Knill, Phys. Rev. Lett. 90, 037901 (2003).

[10] G. Gordon, G. Kurizki, and D. A. Lidar, Phys. Rev. Lett. 101, 010403 (2008).

[11] J. R. West, B. H. Fong, and D. A. Lidar, Phys. Rev. Lett. 104, 130501 (2010).

[12] S. Pasini, P. Karbach, and G. S. Uhrig, arXiv:1009.2638v2.

[13] Liang Hao, Wen Yi Huo, and Gui Lu Long, J. Phys. B: At. Mol. Opt. Phys. 41, 125501 (2008).

[14] A. G. Palmer III, M. J. Grey, C. Wang, Methods in Enzymology, 394, 430 (2005).

[15] G. S. Uhrig Phys. Rev. Lett 98, 100504 (2007).

[16] S. Pasini and G. S. Uhrig, Phys. Rev. A 81, 012309 (2010).

[17] M. J. Biercuk, H. Uys, A. P. VanDevender, N. Shiga, W. M. Itano, and J. J. Bollinger, Nature 458, 996 (2009).

[18] M. J. Biercuk, H. Uys, A. P. VanDevender, N. Shiga, W. M. Itano, and J. J. Bollinger, Phys. Rev. A 79, 062324 (2009).

[19] J. Du, X. Rong, N. Zhao, Y. Wang, J. Yang, and R. B. Liu, Nature 461, 1265 (2009).

[20] G. A. Alvarez, A. Ajoy, X. Peng, and D. Suter, Phys. Rev. A 82, 042306 (2010).

[21] L. Cywinski, R. M. Lutchyn, C. P. Nave, and S. Das Sarma, Phys. Rev. B 77, 174509 (2008).

[22] G. de Lange, Z. H. Wang, D. Riste, V. V. Dobrovitski, and R. Hanson, Science 330, 60 (2010).

[23] C. Barthel, J. Medford, C. M. Marcus, M. P. Hanson, and A. C. Gossard, arXiv:1007.4255 (2010).

[24] C. A. Ryan, J. S. Hodges, and D. G. Cory, Phys. Rev. Lett. 105, 200402 (2010).
[25] Yoav Sagi, Ido Almog, and Nir Davidson, Phys. Rev. Lett. 105, 053201 (2010).

[26] Ashok Ajoy, G. A. Alvarez, and D. Suter, arXiv:quant$\mathrm{ph} / 1011.6243 \mathrm{v} 2$.

[27] G. S. Agarwal, Phys. Scr. 82 (2010) 038103.

[28] Y. Wang, X. Rong, P. Feng, W. Xu, B. Chong, Ji-Hu $\mathrm{Su}$, J. Gong, and J. Du, Phys. Rev. Lett. 106, 040501 (2011).

[29] G. S. Uhrig, Phys. Rev. Lett. 102, 120502 (2009).

[30] J. R. West, B. H. Fong, and D. A. Lidar, Phys. Rev. Lett. 104, 130501 (2010).

[31] S. S. Roy and T. S. Mahesh, J. Magn. Reson. 206, 127 (2010).

[32] S. S. Roy and T. S. Mahesh, Phys. Rev. A 82, 052302 (2010).

[33] D. G. Cory, A. F. Fahmy, and T. F. Havel, Proc. Natl. Acad. Sci. USA 94, 1634 (1997).

[34] G. Pileio, M. Concistr, M. Carravetta, and M. H. Levitt, J. Magn. Reson. 182, 353 (2006).

[35] M. Carravetta and M. H. Levitt, J. Am. Chem. Soc. 126, 6228 (2004).

[36] M. Carravetta, O. G. Johannessen, M. H. Levitt, Phys. Rev. Lett. 92, 153003 (2004).

[37] M. Carravetta and M. H. Levitt, J. Chem. Phys. 122, 214505 (2005).

[38] G. Pileio and M. H. Levitt, J. Chem. Phys. 130 (2009) 214501.

[39] K. Gopalakrishnan and G. Bodenhausen, J. Magn. Reson. 182, 254 (2006).

[40] G. S. Uhrig and Stefano Pasini, Phys. Rev. Lett. New. J. Phys. 12045001 (2010).

[41] S. Pasini, P. Karbach, and G. S. Uhrig, manuscript obtained by private communication. 